\title{
Brief physician advice to problem drinkers resulted in economic benefits
}

Fleming MF, Mundt MP, French MT, et al. Benefit-cost analysis of brief physician advice with problem drinkers in primary care settings. Med Care 2000 Jan;38:7-18.

QUESTION: In patients with drinking problems, is brief advice given by a physician cost effective?

\section{Design}

Cost benefit analysis of a randomised controlled trial with 12 months of follow up.

\section{Setting}

17 community clinics in Wisconsin, USA comprising practices of 64 family physicians and general internists.

\section{Patients}

774 patients who were $18-65$ years of age (62\% men) and drank $>14$ alcoholic drinks/week (>168 g alcohol/wk) (>11 drinks/week for women [ $>132 \mathrm{~g}$ alcohol/wk]*). Exclusion criteria were pregnancy, attendance at an alcohol treatment programme or symptoms of alcohol withdrawal in the previous year, physician advice to change alcohol use in the previous 3 months, consumption of $>50$ drinks/week $(>600 \mathrm{~g}$ alcohol/wk), or symptoms of suicide.

\section{Intervention}

Patients were allocated to a brief physician advice intervention $(n=392)$ or a control group $(n=382)$. The intervention consisted of printed feedback on health behaviours and previous problem drinking, adverse effects of alcohol, drinking cues, and diary cards. Intervention group patients had two 15 minute physician visits 1 month apart and follow up telephone calls. Control group patients received a general health booklet.

\section{Main cost and outcome measures}

Main clinical outcomes were change in alcohol use, healthcare use, and change in alcohol related events. The costs were considered from the perspective of the clinic (equipment and personnel) and patient (lost wages and transportation). The economic benefits pertained to reductions in healthcare use, legal events, and motor vehicle accidents.

\section{Main results}

Patients who received the brief physician intervention had greater reductions in alcohol use than patients in the control group. Intervention group patients reported fewer days of hospitalisation than control group patients $(p=0.046)$ and were involved in fewer motor vehicle accidents and criminal events, but the difference with control group patients was not statistically significant. The total clinic cost (initial screening, assessment, intervention sessions, staff training sessions, and follow up telephone calls) was US\$64 933 (\$165.65 per intervention patient). Patient resource costs (travel and lost work time) were $\$ 15277$ (\$38.97 per patient). The total cost of the intervention was $\$ 80210$ (\$205 per intervention patient). The saving in service utilisation cost was \$195 448 (\$523 per patient). The saving in legal events and motor vehicle accidents was \$228 071 (\$629 per patient). The total benefit of the brief intervention was $\$ 423519$ (\$1151 per patient) $(\mathrm{p}=0.009)$. The net benefit per patient was $\$ 947$. The benefit cost ratio was $\$ 56263$ for every $\$ 10000$ invested.

\section{Conclusions}

In patients with drinking problems, brief advice given by a physician was cost effective for both patients and the healthcare system. The net benefit per patient was US $\$ 947$.

$* 1$ drink $=1.5$ units of alcohol.
Sources of funding: Robert Wood Johnson Foundation; National Institute on Drug Abuse; National Institute on Alcohol Abuse and Alcoholism.

For correspondence: Dr M F Fleming, Center for Addiction Research and Education, University of Wisconsin-Madison, 777 South Mills Street, Madison, WI 53715 , USA.Fax +1608263 5813.

Abstract and commentary also appear in ACP Journal Club and Evidence-Based Medicine.

\section{COMMENTARY}

The study by Fleming et al gives further evidence that early intervention with non-dependent heavy drinkers is effective in changing drinking behaviour. Patient selection by opportunistic screening, and the simplicity of the intervention make this study particularly attractive for primary care. The mean level of consumption would hardly raise eyebrows in normal practice, and might result in a brief mention of recommended limits. This is basically what the control group receives. The study shows that a structured approach, focused around individual patients and their drinking patterns, is much more effective.

The study presents a robust attempt at calculating a financial cost benefit analysis for the health intervention. The use of "opportunity cost" is germane to the real world of medicine, where questions revolve around the allocation of limited resources. The conclusion that resource allocation to primary prevention produces major overall resource savings, particularly in secondary care, will be of great relevance to total healthcare purchasers or providers such as primary care trusts. The benefits are not restricted to the healthcare system. Accidents, injuries, and crime were all shown to decrease after the intervention, although some of the variables failed to reach statistical significance when taken individually. This is not just an intervention that saves hospitals money, it also improves the patients' lives, and makes society a safer place!

Some questions arise from a lack of detail. We do not know the take up rate of the intervention or the number of patients lost to follow up. There is no indication about whether intervention group patients visited their primary care physicians less, which would be an obvious benefit to those physicians delivering the intervention. Some breakdown of the benefits by level of consumption would be useful. Is there any benefit in targeting the group drinking just over "safe" limits? The figures showing healthcare use also suggest that the benefit may not be sustained at the 12 month follow up point, and it would be interesting to know whether the effects of the intervention continue, or whether further "top up" intervention is required.

Pete Sudbury, MRCPsych, MBA Heatherwood and Wexham Park Hospitals Trust Slough, Berkshire, UK 